\title{
Secondary Knee Osteoarthritis with Medial Instability and Tibial Defect due to Chronic Abscess Treated by Total Knee Arthroplasty with Metal Augmentation - Case Report
}

\author{
Bintang Soetjahjo, Udi Herunefi Hancoro, Rieva Ermawan, \\ Rhyan Darma Saputra, Hillan Akbar
}

\author{
Department of Orthopaedic \& Traumatology Faculty of Medicine, Universitas Sebelas Maret/ \\ Dr. Moewardi General Hospital/ Prof. Dr. R. Soeharso Orthopaedic Hospital, Surakarta
}

\section{ABSTRACT}

Background:Knee osteoarthritis (OA), known as degenerative joint disease, is typically the result of wear and tear and progressive loss of articular cartilage, most common in elderly women and men. Knee OA is a leading cause of disability and increase with an aging and overweight population. Total Knee Arthroplasty (TKA) is a common surgical treatment of OA knee that highly cost-effective procedure. Stable placement of components is difficult and thus it presents technical challenges for the surgeon but various options are available for managing these defects.

Case Report: A 58 years-old housewife came to our clinic due to chronic left knee pain with associated instability since two years ago and getting worse in last three months. She already had knee joint supplementation injections and physical therapy but no improvements. Based on our clinical and radiological findings, our assessment was secondary left knee osteoarthritis with medial instability and tibial defect due to chronic abscess. Patient underwent TKA to correct this condition. A revised implant was used to close the defect in the medial part of the tibia and soft tissue reconstruction procedure was used to correct the instability.
Result: On the first postoperative month, the patient underwent rehabilitation and physical therapy to strengthen her lower limb muscles. On the second month, she started to walk using walking assisted device. On the ninth months follow-up, the patient was able to walk normally without assisted device, the pain disappeared and the symptoms of knee instability were reduced.

Conclusion:Bone defects are commonly encountered during TKA, which still has no standard treatment to cure. In this case, the use of metal augmentation with revision implant give satisfactory results with good clinical outcome.

Keywords: Osteoarthritis, Total Knee Arthroplasty, knee instability, tibial defect, metal augmentation

\section{Correspondence:}

Bintang Soetjahjo. Dr. Moewardi Hospital. Jl. Kolonel Sutarto 132, Jebres, Surakarta, Central Java 57126, Indonesia. Mobile: +628122987359. Email: bjortho@yahoo.com

Cite this as:

Soetjahjo B, Hancoro UH, Ermawan R, Saputra RD, Akbar H (2021). Secondary Knee Osteoarthritis with Medial Instability and Tibial Defect due to Chronic Abscess Treated by Total Knee Arthroplasty with Metal Augmentation - Case Report. Indones J Med. 06(02): 168-176. https://doi.org/10.26911/theijmed.2021.06.02.06.

(i) (2) Indonesian Journal of Medicine is licensed under a Creative Commons cc)

\section{BACKGROUND}

Knee osteoarthritis (OA), also known as degenerative joint disease, is typically the result of wear and tear and progressive loss of articular cartilage. It is most common in elderly women and men. Knee OA can be divided into two types, primary and secondary. Primary osteoarthritis is arti- 
cular degeneration without any apparent underlying reason. Secondary osteoarthritis is the consequence of either an abnormal concentration of force across the joint as with post-traumatic causes or abnormal articular cartilage (Elsiwy et al., 2019; Nilsson et al., 2018; Springer, 2019).

Currently, the lifetime risk of developing symptomatic knee $\mathrm{OA}$ is approximately 50\% (Murphy et al., 2008). Knee OA is a leading cause of disability in persons in the developed countries and this is only projected to increase with aging and overweight population (Zeni and Mackler, 2010). Pain is the leading symptom of OA and often chronic in nature, leading to significant morbidity and decreased quality of life (Abou-Raya et al., 2012). Knee osteoarthritis commonly requires joint replacement, substantially reduces quality of life and increases health care utilization and costs (Eckstein et al., 2013).

Treatment for knee OA begins with conservative methods and progresses to surgical treatment options when conservative treatment fails (Elsiwy et al., 2019; Nilsson et al., 2018; Springer, 2019). Total Knee Arthroplasty (TKA) is a common surgical treatment of $\mathrm{OA}$ in the knee. Knee arthroplasty is a surgical procedure undertaken to replace the weight-bearing surfaces of the knee joint to restore capacity and function (Werner et al., 2003). It can be performed as a partial, also called unicompartmental arthroplasty (UKA, which replaces only the damaged surfaces retaining any undamaged parts) or a total knee arthroplasty (TKA) (Ji et al., 2014). TKA involves replacement of all three compartments of the knee, known as the medial compartment (inside aspect of the knee), the lateral compartment (outside of the knee), and the patellofemoral compartment (the joint between the patella and the femur) (Rytter et al., 2009). TKA is one of the most common and reliable surgical treatment options for the treatment of knee diseases such as rheumatoid arthritis, osteoarthritis, and osteonecrosis (Van Manen et al., 2012). The goal of TKA is to reduce pain, enhance functional capacity, and improve health-related quality of life and life expectancy (Chang et al., 2014)

TKA is a highly cost-effective procedure and the incidence of TKA has increased dramatically in the aging population with knee arthritis over the past few decades. As the population ages and the use of total knee arthroplasty (TKA) expands, peripheral bone defects of the medial tibia are frequently encountered in primary total knee arthroplasty (TKA) for varus knees (Tsukada et al., 2013). Stable placement of components is difficult in these cases and thus presents technical challenges for the surgeon. Various options are available for managing these defects. Countermeasures for bone defects include increased bone resection, lateralizing the tibial component, cement filling, bone grafting, metal augmentation, and the use of custom-made prostheses (Cuckler, 2004). We report the case of a 58-year-old female patient with secondary OA knee with medial instability and tibial defect treated by Total Knee Arthroplasty with metal augmentation.

\section{CASE REPORT}

A 58 years-old housewife came to our clinic with chief complain pain on her left knee that was felt since two years ago and getting worse in last three months. The pain was aggravated by movement and by walking. Swelling at the knee also has been reported by the patient. The patient has undergone knee joint supplementation injections in our outpatient clinic, and also physiotherapy since the last six months. The patient can walk without assisted devices but only for 50 meters far due to the knee 
pain. From past medical history, the patient was obese and had type II diabetes controlled with Metformin medication and Insulin injection. The patient also had a debridement surgery for an abscess on his left thigh 3 years ago. There was neither history of other diseases or trauma.

From physical examination of the left knee seen in figure 1, there was three surgical scars on the anterior, medial and lateral sides. Swelling and severe varus deformity was found in the patient's left knee. No fistulas and no sign of inflamation

were found. From palpation examination, crepitation was found when the patient's knee was flexed and extended, but there was no tenderness nor neurovascular disturbances. Examination of the knee's active and passive Range of Movement (ROM) was 10 degrees hyperextension and 100 degrees flexion. Patello Femoral Grind Test was positive at bilateral side, Anterior and posterior drawer test of left knee was negative, with positive grade III of varus stress test.
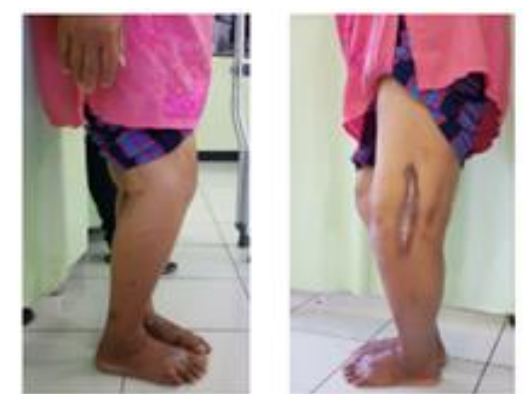

Figure 1. Pre-operative appearance of the patient's knee.

From left knee X-ray seen in figure 2, there were obvious signs of narrowing knee joint space, marginal osteophytes, subchondral sclerosis and marked varus deformity, or what we usually called as Cardinal Signs of knee Osteoarthritis (OA).
The Tibio-femoral Angle was varus 24 degrees, and bony defect was seen at tibial medial condyle with subluxation. From laboratoy examination the HbA1c level was 9.6\% with other laboratory findings were within normal range.

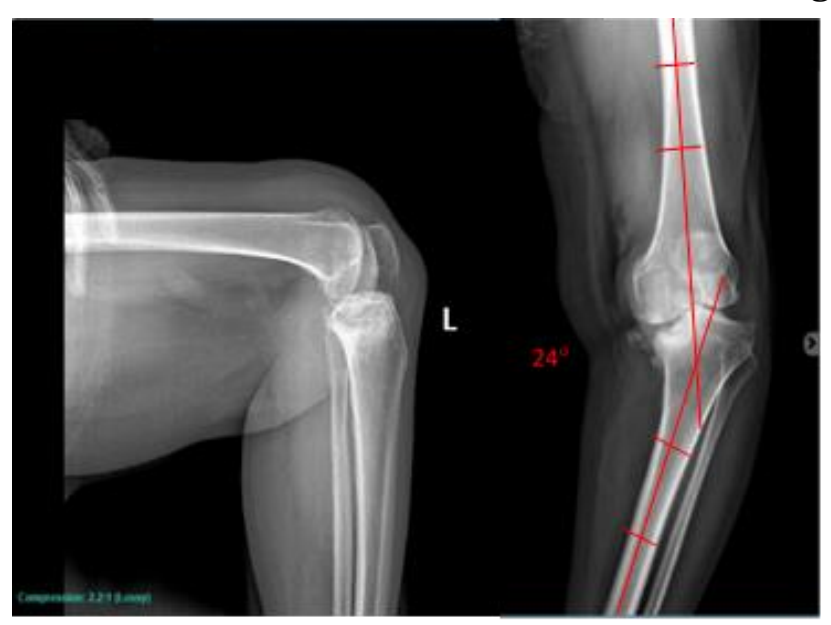

Figure 2. Pre-Operative X-ray

Based on the clinical and radiological findings, our assessment was left knee osteoarthritis with severe varus deformity, Kellgren-Lawrence classification grade IV. 
The goals of our treatments were to achieve painless, stable and functional knee joint. We planned to performed left Total Knee Arthroplasty with additional metal block augmentation to compensate the proximal tibial defect and knee ligament laxity.

The surgical technique of using modular metal blocks or wedges is relatively straightforward. Once the location and extent of the defects have been determined, the size and shape of the augment that best fits that defect is selected. It is helpful to use intramedullary instrumentation to align the tibial cut perpendicular to the mechanical axis of the tibia and the associated cutting guide will guide the 1 - to 2-mm "skim" or "freshening" cut on each plateau to enact the least amount of bone removal, and remove bone to accept the exact size and shape of the augment. It is also import- ant to determine the proper tibial component rotation, which is typically aligned with the medial one-third of the tibial tubercle, so that the sagittal cut of the block augment will seat in the corresponding correct rotational position. Once the cuts are made, sclerotic bone is roughened to facilitate cement interdigitation and the final tibial component with stem extension is placed.

Femoral osteotomy was performed using an intramedullary rod. The distal femoral osteotomy was aimed to be performed in valgus angulation equal to the angle between the anatomical and functional axes of the femur and in external rotation parallel to the epicondylar axis. Intraoperatively we did not found any unhealthy tissue around the knee joint.
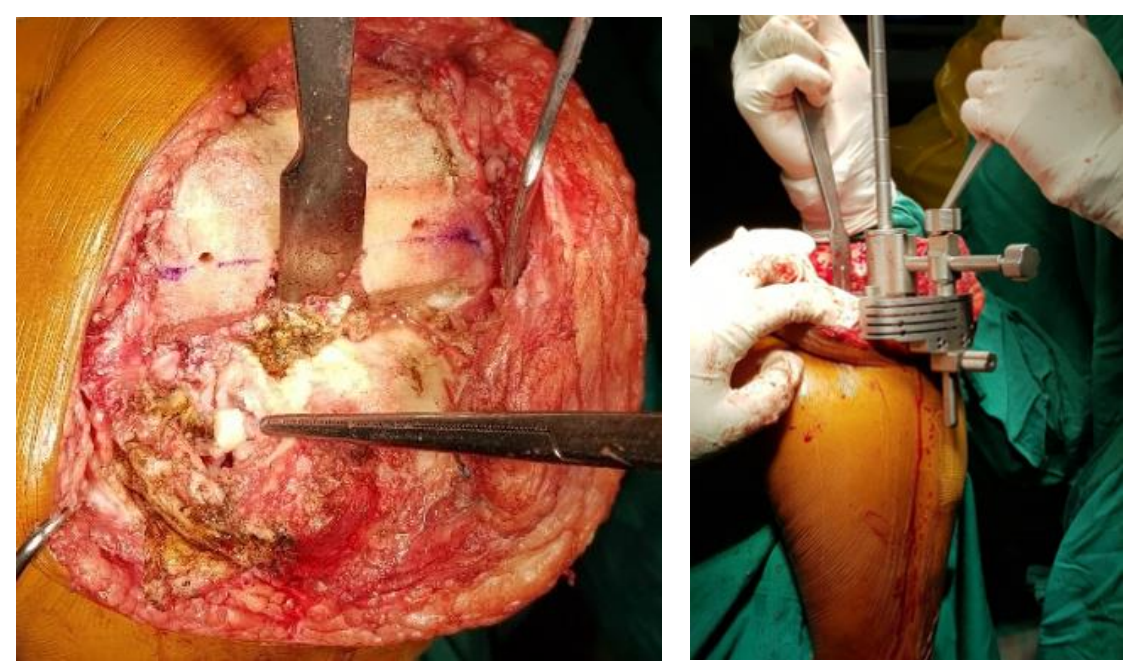

Figure 3. (A) Intra-Operative findings of moderate medial tibia defect.

(B) Reconstrustion of the tibial platform and measuring the thickness of metal augmentation.

After the total knee arthroplasty was performed, the patient's varus deformity was corrected with a tibio-femoral angle of 4 degrees valgus. On the first postoperative month, the patient underwent rehabilitation and physical therapy to strengthen her lower limb muscles. On the second month, she started to walk using walking assisted device. On the ninth months follow-up, the patient was able to walk normally without assisted device, the pain disappeared and the symptoms of knee instability were reduced. The functional outcome was good and the goals of treatment were achieved. 


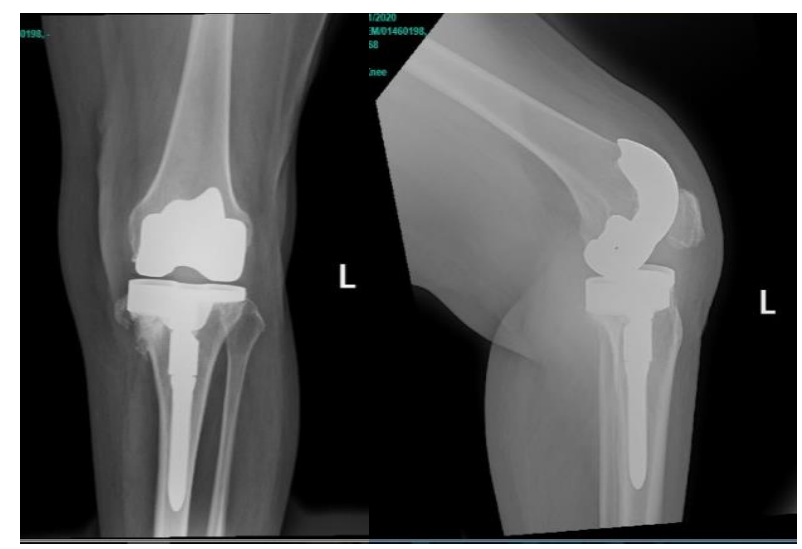

Figure 4. Post Operative X-ray

\section{DISCUSSION}

The study carried out by Manusco et al in 1996 suggested that there is no clear indication for TKR. As the most common reasons are severe pain and disability score, radiological evidence, and moreover on the agreement of patients to undergo TKR (Wood et al., 2009). This can be combined with financial security and assistance due to high cost for surgery, access to healthcare facilities in addition to the patient's awareness and literacy rate. However, this has been according to the viewpoint of orthopaedic surgeons, but the data based on patients need to undertake TKR is not known (Mancuso et al., 1996). In the study conducted by Furtado et al found that the commonest indication for TKR was OA with 93.58\% frequency and the next being rheumatoid arthritis (RA) with a highly significant correlation. The reason for increased incidence of TKR surgeries in the recent times as compared to the past can be due to increased awareness among people relating to its benefits (Furtado et al., 2016).

Furtado et al also found that when comparing diabetes and OA prevalence, the correlation between them was very strong (Furtado et al., 2016). A study performed involving rats stated that there was a higher incidence of osteoarthritis noticed in type 2DM rats as compared to controls along with Cysts formation at the junction of the articular cartilage and subchondral bone (Onur et al., 2014). There is evidence of biological link existing between bone loss at subchondral bone plate in knee OA and hypertension and T2DM (Wen et al., 2013). Thus, secondary OA that cause tibial defect in this case had correlation with T2DM.

The etiology of bone defects in primary TKR include erosion secondary to arthritic change, trauma, osteonecrosis, and a previous osteotomy. The defects typically have a condensed sclerotic surface. This contrasts with what is observed at revision surgery, where following removal of a component an osteopenic surface is revealed. Varus deformities are characteristically associated with medial defects (Lee and Choi, 2011). In this case chronic abscess due to uncontrolled T2DM was main reason the patient had bone defect.

Various techniques have been developed to manage the bone defects in RTKA, including cementation, modular metal augments, elimination of bone defects, bone grafts (autografts, allografts and structural massive bone allografts), metaphyseal fixation (porous titanium metaphyseal sleeves and porous tantalum metaphyseal cones), and megaprostheses (customized prostheses) (Huten, 2013; Qiu et al., 2012). Whereas determine best techniques for 
used depends on tibial bone defect classification. According to the AORI (Anderson Orthopedic Research Institute) classification, bone defects are classified into three types for tibia ( $\left.\mathrm{T} 1, \mathrm{~T} 2, \mathrm{~T}_{3}\right)$. In type 1 ( $\mathrm{T} 1$ ) defects, there is a minor bone defect without compromising the stability of a revision component. The development of posterior condyles remains normal. In type 2 (T2) defects, metaphyseal bone damage and cancellous bone loss occurred in one femoral condyle/tibial plateau (type 2A: T2A) or both femoral condyle/tibial plateau (type 2B: T2B). The development of the posterior condyles and/or tibial component is reduced. In type 3 (T3) defects, there is significant cancellous metaphyseal bone loss compromising the ligamentous instability of a major portion of the condyle or plateau (Huten, 2013; Qiu et al., 2011).

In this case we used metal augmentation to correct large tibial defect on patient. Modular metal augmentation provides more stable and durable knee revisions with limited peripheral bone defects up to 20-mm deep. Various types of augments (such as rectangular blocks and wedges) are available that allow selective augmentation for both femoral and tibial defects (Qiu et al., 2012). The preference between wedges and blocks depends on the shape of bone defects. The augment that best fills the defect and, at the same time, removes little intact host bone should be used. Augments are screwed or cemented to fit the femoral and tibial components (Huten, 2013). On the tibial side, modular metal wedges are usually used to augment the tibial tray for tibial bone stock deficiency. Femoral defects most often occur on the posterior surfaces and metal blocks can be used to increase femoral component rotation and to maintain the balance between flexion and extension gap. This technique should be reserved for elderly and less active patients
(Huten, 2013; Qiu et al., 2012). Metal augments confer the risk of some complications, including fretting, radiolucent lines and corrosion. It has also been reported that the metal augmentation technique may also cause the disassociation of modular components, leading to stress shielding and increased potential bone loss (Patel et al., 2004). Werle et al. suggest that metal augmentation is an acceptable technique (Werle et al., 2002). In their study, they used large $(30 \mathrm{~mm})$ metal distal femoral augments to compensate for type 3 bone defects and observed no radiographic evidence of loosening; no implants had been revised after a mean of 37 months. Patel et al. treated a total of 102 RTKA patients (type 2 defects) with metal augments and observed $92 \%$ survival at 11 years, with no significant complications, including fretting and loosening (29). Lee et al. followed up 37 patients (39 knees) for more than 2 years after revision of infected TKA using modular metal augments for bone defects and concluded that increased modularity can result in radiological stability (Lee et al., 2016).

In the current series, a revised implant is used to close the defect in the medial part of the tibia. The clinical results obtained, the patient was able to walk normally, the pain disappeared and the symptoms of medial instability were reduced.

Bone defects are commonly encountered during TKA, which still has no standard treatment to cure. Only management of femoral and tibial bone defects is possible in some cases. The preference between different management systems depends on the patients and types of bone defects. Accurate diagnosis of bone defects and proper selection of treatment methods are necessary to improve the survival rate and construction stability. In this case, the 
use of metal augmentation with revision implant give satisfactory results with clinical outcome patient was able to walk normally, the pain disappeared and the symptoms of medial instability were reduced.

\section{AUTHOR CONTRIBUTION}

Each author individually made significant contributions to the development of this manuscript.

\section{CONFLICT OF INTEREST}

All the authors declare that there was no conflict of interest referring to this manuscript.

\section{FUNDING AND SPONSORSHIP}

No financial support has been received from any source.

\section{ACKNOWLEDGEMENT}

We would like to thank Dr. Moewardi Hospital, Surakarta, Central Java, and the patients, for giving permission to use the data to this study.

\section{REFERENCE}

Chang MJ, Lim H, Lee, NR, Moon, YW (2014). Diagnosis, causes and treatments of instability following total knee arthroplasty. Knee Surg. Relat. Res. 26(2): 61-67. https://doi.org/10$.5792 / \mathrm{ksrr} .2014 .26 .2 .61$.

Cuckler JM (2004). Bone loss in total knee arthroplasty: Graft augment and options. J Arthroplasty. 19(4 Suppl 1): 56-58. https://doi.org/10.1016/j.arth.2004.03.002.

Eckstein F, Kwoh CK, Boudreau RM, Wang Z, Hannon MJ, Cotofana S, Hudelmaier MI, et al. (2013). Quantitative MRI measures of cartilage predict knee replacement: A case-control study from the Osteoarthritis Initia- tive. Ann Rheum Dis. 72(5): 707-714. https://doi.org/10.1136/annrheumdis -2011-201164.

Elsiwy Y, Jovanovic I, Doma K, Hazratwala $\mathrm{K}$, Letson $\mathrm{H}$ (2019). Risk factors associated with cardiac complication after total joint arthroplasty of the hip and knee: A systematic review. J Orthop Surg Res. 14(1): 15. https://doi.org/10.1186/s13018-018-1058-9.

Furtado C, Deshpande M, Dias A (2016). Clinical profile of patients undergoing total knee replacement (TKR)-case based series. IOSR J Dent Med Sci. 15(1): 95-101. https://doi.org/10.979o/o853-151395101.

Huten D (2013). Femorotibial bone loss during revision total knee arthroplasty. Orthop Traumatol Surg Res. 99(1): S22-S33. https://doi.org/10.1016/j.otsr.2012.11.009.

Ji JH, Park SE, Song IS, Kang H, Ha JY, Jeong JJ (2014). Complications of medial unicompartmental knee arthroplasty. Clin Orthop Surg. 6(4): 365-372. https://doi.org/10.4055/cios.2014.6.4.365.

Lee JK, Choi CH (2011). Management of tibial bone defects with metal augmentation in primary total knee replacement. J Bone Joint Surg Br. 93(11): 1493-1496. https://doi.org/10.1302/0301-620x.93b10.27136.

Lee KJ, Bae KC, Cho CH, Son ES, Jung JW (2016). Radiological stability after revision of infected total knee arthroplasty using modular metal augments. Knee Surg Relat Res. 28(1): 55-61. https://doi.org/10.5792/ksrr.2016.28 .1 .55 .

Mancuso CA, Ranawat CS, Esdaile JM, Johanson NA, Charlson ME (1996). Indications for total hip and total knee arthroplasties: Results of orthopaedic surveys. J Arthroplasty. 11(1): 
Soetjahjo et al./ Total Knee Arthroplasty with Metal Augmentation

34-46. https://doi.org/10.1016/so883-5403(96)80159-8.

Manen MDV, Nace J, Mont, MA (2012). Management of primary knee osteoarthritis and indications for total knee arthroplasty for general practitioners. J Am Osteopat. Assoc. 112(11): 709715. https://doi.org/10.7556/jaoa.2012.112.11.709.

Murphy L, Schwartz TA, Helmick CG, Renner JB, Tudor G, Koch G, Dragomir A, et al. (2008). Lifetime risk of symptomatic knee osteoarthritis. Arthritis Care Res. 59(9): 1207-1213. https://doi.org/10.1002/art.24021.

Nilsson ÅL Dencker A, Palstam A, Person G, Horton MC, Escorpizo R, Küçükdeveci AA, et al. (2018). Patientreported outcome measures in osteoarthritis: A systematic search and review of their use and psychometric properties. RMD Open. 4(2): eoo0715. https://doi.org/10.1136/rmdopen-2018-000715.

Onur T, Wu R, Metz L, Dang A (2014). Characterisation of osteoarthritis in a small animal model of type 2 diabetes mellitus. Bone Joint Res. 3(6): 203211. doi.org/10.1302/2046-3758.36.2000244.

Patel JV, Masonis JL, Guerin J, Bourne RB, Rorabeck CH (2004). The fate of augments to treat-type-2 bone defects in revision knee arthroplasty. J Bone Joint Surg Br. 86(2): 195-199. https://doi.org/10.1302/0301-620x.86b2.13564.

Qiu YY, Yan CH, Chiu KY, Ng FY (2011). Review article: Bone defect classifications in revision total knee arthroplasty. J Orthop Surg. (Hong Kong). 19(2): 238-43. https://doi.org/10.1177/230949901101900223.

Qiu YY, Yan CH, Chiu KY, Ng FY (2012). Review article: Treatments for bone loss in revision total knee arthroplasty. J Orthop Surg (Hong Kong). 20(1): 78-86. https://doi.org/10.1177/230949901202000116.

Raya SA, Raya AA, Helmii M (2012). Duloxetine for the management of pain in older adults with knee osteoarthritis: Randomised placebo-controlled trial. Age Ageing. 41(5): 646-652. https://doi.org/10.1093/ageing/afso72.

Rytter S, Egund N, Jensen LK, Bonde JP (2009). Occupational kneeling and radiographic tibiofemoral and patellafemoral osteoarthritis. J Occup Med Toxicol. 4: 19. https://dx.doi.org/10.1186\%2F1745-6673-4-19.

Springer BD (2019). Management of the Bariatric Patient. What are the implications of obesity and total joint arthroplasty: The Orthopedic Surgeon's Perspective?. J Arthroplasty. 34(7S): S30-S32. https://doi.org/10.1016/j.arth.2018.12.021.

Tsukada S, Wakui M, Matsueda M (2013). Metal block augmentation for bone defects of the medial tibia during primary total knee arthroplasty. J Orthop Surg Res. 8: 36. https://doi.org/10.1186/1749-799x-8-36.

Wen CY, Chen Y, Tang HL, Yan CH, Lu WW, Chiu KY (2013). Bone loss at subchondral plate in knee osteoarthritis patients with hypertension and type 2 diabetes mellitus. Osteoarthritis Cartilage. 21(11): 1716-23. https://doi.org/10.1016/j.joca.2013.06.027.

Werle JR, Goodman SB, Imrie SN (2002). Revision total knee arthroplasty using large distal femoral augments for severe metaphyseal bone deficiency: A preliminary study. Orthopedics. 25 (3): 325-7. https://doi.org/10.3928/0147-7447-20020301-17.

Werner A, Jäger M, Schmitz H, Krauspe R (2003). Joint preserving surgery for 
Soetjahjo et al./ Total Knee Arthroplasty with Metal Augmentation

osteonecrosis and osteochondral defects after chemotherapy in childhood. Klin Padiatr. 215(6): 332-337. https://doi.org/10.1055/s-2003-45495.

Wood GC, Naudie DDR, MacDonald SJ, McCalden RW, Bourne RB (2009). Results of press-fit stems in revision knee Arthroplasties. Clin Orthop
Relat Res. 467(3): 810-817. https://doi.org/10.1007/s11999-008-0621-9.

Zeni JA, Mackler LS (2010). Clinical outcomes after simultaneous bilateral total knee arthroplasty. Comparison to unilateral total knee arthroplasty and healthy controls. J Arthroplasty. 25(4): 541-546. https://doi.org/10.1016/j.arth.2009.02.016. 